\title{
Effects of Resting Actions Using Smart Toys During Break Times on Concentration in E-learning
}

\author{
Takashi Ito $^{1}$, Kenichi Takahashi ${ }^{*}, 2$, Tomoko Kajiyama ${ }^{2}$ \\ ${ }^{1}$ Aoyama Gakuin University, Department, Institute of Industrial and Systems Engineering, Kanagawa, 252-5258, Japan \\ ${ }^{2}$ Hiroshima City University, Department of Intelligent Systems, Hiroshima, 731-3194, Japan
}

\begin{tabular}{l} 
A R T I C L E I N F O \\
\hline Article history: \\
Received: 24 August, 2020 \\
Accepted: 15 October, 2020 \\
Online: 22 October, 2020
\end{tabular}

Keywords:

Refresh

Resting action

Smart toys

Effect

Break times

\begin{abstract}
A B S T R A C T
This study conducted experiments to investigate the effects of resting actions of e-learners during break times on keeping and improving the concentrations of learners in e-learning. Two smart toys (a dog-type robot "aibo" and a toy drone) were used for the resting actions. Two types of experiments were conducted to examine the effects: one is a work type experiment (simple mathematical calculation) and the other is a memorizing type experiment (learning of English words). In those experiments, the learners played with one of the smart toys during the resting times between the learning sessions and performed certain actions accordingly. The experimental results were combined with the previous study using a humanoid robot "RoBoHoN." A questionnaire was also employed to investigate the feeling of the learners. The experiments showed that the "playing with aibo" action refreshed the learners effectively in the mathematical experiment, while the "playing with RoBoHoN" action was effective in the learning experiment.
\end{abstract}

\section{Introduction}

In recent years, e-learning has been actively used with the development of ICT [1]. E-learning is the education and learning way that combines the Internet with PCs and mobile terminals. With e-learning, learners can deeply understand the content by learning iteratively and utilizing videos and voices. Also, learners can take advantage of good features of e-learning, such as learning at any time and place and furnishing functions for learners to select learning contents suitable to their knowledge level and to repeat lessons, as well as the variety of devices used for e-learning, such as tablets and smartphones. As a result, learning effect by elearning is expected to be higher than the conventional learning using paper media. Additionally, with e-learning systems, the management and utilization of learning data become easy.

On the other hand, since e-learning is mainly used for individual learning, there is no instructor present and there is no opportunity that learners communicate with instructors and other students. The stimulation from instructors and friends is small, which can decrease their willingness to learn gradually. Thus, it is not easy for learners to keep motivation and concentration high, and learners can easily get bored to abandon learning, which is one of the important problems to be solved for e-learning [2].

\footnotetext{
* Corresponding Author: Kenichi Takahashi, Hiroshima City University, +81-82848-2872,6961643@hiroshima-cu.ac.jp
}

In order to challenge the problem, various systems have been proposed, e.g., a system with a function to praise and scold students with images according to their learning performance, a system that measures student concentration and learning states during learning using Kinect, a system introducing game-based learning, intelligent collaborative e-learning systems, an e-learning platform, and portfolio assessment in elearning environment [3-6].

Another important factor is to provide appropriate break times in order to maintain motivation and concentration of learners. A study investigated the effect on student learning performance by introducing a break during a 90-minute lecture at a university [7]. It showed that taking a short break of about 8-10 minutes improved the learning performance: After the break, drowsiness of students decreased and their concentration increased. In addition, for VDT (visual display terminal) work, a study examined the lengths of an appropriate work time and the break time in order to reduce the burden on the mind and body and to improve work performance [8]. They suggested that one session of continuously working on a VDT is less than one hour and a 1015 minute break between working sessions is desirable. Also, work on the way how office workers using VDTs spent their break times influenced their working efficiency was published [9], and Japan's Ministry of Health, Labor and Welfare recommends occupational health management guidelines regarding the use of 
VDTs [10]. Further, apps for supporting learners in the break time has been proposed [11]. The authors consider that it is important to introduce appropriate resting actions during break times for efficient and effective learning in e-learning that usually uses the VDT equipment.

In our research, a web-based e-learning system equipped with functions to improve or maintain learning motivation and concentration has been developed, such as a ranking function, a function that praises or scolds students appropriately with images and a sound, and a function for learners to present problems to one another [3]. In addition, a learning system equipped with games to investigate the effect of fun and relaxation of the games has been developed [12]. The games are one that refreshes learners by quick physical motion (shooting flies on a screen) and one that relaxes learners by taking care of a dog without heavy physical movement. These studies showed that refreshing by playing games during the break times between learning sessions is effective on improving learners' will to learn and maintaining the degree of concentration during e-learning. Furthermore, to examine what kinds of resting actions during break times keep or increase the concentration, we have conducted mathematical calculation experiments and English word memorizing experiments previously [13], [14]. The resting actions(behaviors) during the break times are: "playing the beatfly game," "playing the dog-care game," "reading a book," "listening to music," and "doing nothing." We have showed that the type of these resting actions influences the degree of concentration.

Additionally, there was a study that introduced new resting actions and investigated the influence of the learner's motivation for learning and concentration. In this study, we performed the same experiments using two types of works as in our previous study: "simple work" (the learners solve continuously simple numeric calculation with four arithmetic operations for 15 minutes per work session) and "learning work" (they memorize English words for 30 minutes per learning session). In the study, we introduced the interaction with communication robots, "RoBoHoN" and "aibo", as new resting actions [15]. The study confirmed that the break time using "playing with aibo" had a good relaxing effect, and had a positive effect on simple learning such as numeric calculation with arithmetic operations. However, it is not appropriate for memorization learning such as English word learning, while a break time using "playing with RoBoHoN" gave better results.

In this study, we employ resting actions using a "toy drone" and aibo, and investigate how they affect the learning motivation, concentration, and learning results. The resting action using aibo in this study was the same as that in the previous study [15]. We also conducted two types of experiments for e-learning: simple work in which students try to calculate the numeric questions of four arithmetic operations continuously for 15 minutes and memorizing in which students try to remember new English words for 30 minutes. After the experiments, the learners were asked to answer a questionnaire and respond to statements, in order to examine how they felt the effect the resting actions had on their concentration in the experiments. We discuss the effect of the resting actions after combining these experimental results with the results in our previous study [15].

\section{Smart Toys}

Recently with the development of artificial intelligence technology, various robots become popular. For example, a robot can make dialogue and conversation with humans. We call those robots smart toys. As these smart toys become popular, they are used for children's learning, while the privacy and security problems occurs [16-18].

This study employed smart toys in order to refresh the feeling of the learners by the resting actions. In the experiments, the learners perform different types of resting actions during break times: "playing with a toy drone" (in which the learners manipulate a toy drone to finish a toy course), "playing with RoBoHoN" (in which the learners play five kinds of games with a humanoid robot), and "playing with aibo" (in which the learners play with a dog-shaped robot by talking and touching).

\subsection{RoBoHoN}

"RoBoHoN" is a fourth-generation mobile communication robot developed by Sharp Corporation [19]. RoBoHoN works as a smartphone and has functions to talk with users and to make actions through the original apps. In our experiments, using software called "Scratch Pack for RoBoHoN," four actions were programmed so that the learners can play with RoBoHoN during break times. We refer to these actions together as "playing with RoBoHoN."

The four actions used for RoBoHoN are as follows.

1) Raise a flag: RoBoHoN speaks a command such as "raise a red (or white) flag", and the learner tries to obey the command correctly.

2) Answer a riddle: RoBoHoN speaks randomly one of five programmed riddles, and the leaner tries to answer it.

3) Guess who-I-am: RoBoHoN pretends to be a thing, a person, or an animal, and gives hints, such as "I am a humanoid". The learner guesses what or who RoBoHoN is from those hints spoken by RoBoHoN. The game continues giving other hints until the learner hits the correct answer or the number of hints becomes a certain number.

4) Dance: RoBoHoN can dance randomly one of more than 20 dances with music.

\section{2. aibo}

A pet robot "aibo" is developed by Sony Corporation [20]. It can walk on four legs and behaves like a puppy. It can recognize persons and changes its behavior gradually as the person interacts it frequently. The latest version of "aibo" employs artificial intelligence techniques and network functions such as expressing emotions and online backup of data. The first "aibo" was developed in 1999, and now new "aibo" developed in 2017 was on sale. With the introduction of artificial intelligence, the technology has improved the robot to show more puppy-like gestures and reactions.

In our previous research, we conducted experiments based on the idea that it will be effective to interact with "aibo", a robot that imitates a puppy, as well as to interact with actual small animals, since a certain improvement effect on e-learning was obtained [21]. In this study, we also adopted "aibo" for one of the resting actions from the same viewpoint. We asked the learners to interact with "aibo" during the break times between sessions. "aibo" performs various actions (behaviors) in response to human words. Specific keywords cause "aibo" to behave as if a dog behaves such 
as "hands" and "sitting", and unique behaviors such as "dance" and "high touch". In addition, there are a special ball and a bone toy that can be used for play. "aibo" can learn tricks gradually by being praised with the words "good" or "good dog" and by being stroked in the part of the head, chin, and the back of its body.

\subsection{Toy Drone}

A drone refers to a remote-controlled or autonomous unmanned aerial vehicle. In the present, it is actively utilized for industrial use in various scenes.

Toy drones are inexpensive to buy, often have a small and lightweight body, and are simple to operate. With these features, even a beginner can easily operate the drone. The toy drone "Tello" developed by Ryze Technology Co. was used in this research [22]. We can use a smartphone as a controller to operate it by connecting the dedicated app to the drone. It can also be operated by connecting the app to a dedicated controller via Bluetooth. A small camera mounted on the front of the drone constantly captures the scenes in front of the drone, which are displayed in real time on the screen of the smartphone. The flight mode can be selected by the app. Acrobatic flight and various aerial photography functions can be easily executed.

In this study, we adopted a resting action called "playing with a toy drone" for comparison with the resting actions of different types of smart toys: "aibo" and "RoBoHoN." They were used for resting to refresh the mood by communicating with the robot. On the other hand, the "playing with a toy drone" action presents "fun" by performing precise operations of the drone, which requires concentration.

We asked the learners to operate the toy drone during the break times between work or learning sessions. A dedicated controller linked to the app is used to operate the drone. We can operate the drone more easily by linking the app with the controller. In order to enable the learners to operate the drone in the 3-minute break time, we completed various connections between the app and the controller in advance. In addition, before the experiments, we distributed the document summarizing how to operate the drone to the learners. Since the experiments were conducted indoors, flight mode functions were limited by eliminating dangerous acrobatic flights.

We constructed a drone course to have the learners perform precise operations. A gimmick of arches, a tunnel, and a slalom were placed for the drone to finish the course in about 1 to 2 minutes per lap. As for the arches, we prepared two patterns: one with multiple arches placed in curve side by side, and the other with multiple arches placed in different altitudes on the floor and a chair. The tunnel was made with multiple arches placed at narrow intervals in the same altitude. The slalom was made of rods of different lengths so that the drone goes forward in zigzags avoiding rods. Figure 1 shows the course used for the experiment. The course shown in Figure 1 is a basic form. We changed the difficulty of the course by changing the layout if the learner desired.

\section{Experiments Incorporating Resting Actions}

In this Section, we describe the results of the mathematical experiment and the English-word learning experiment, and discuss how the different types of resting actions affect learning motivation and maintenance of concentration. We estimate the effects of the resting actions on simple work (mathematical calculation) and learning work (memorizing English words) through the experiments. In the experiments, ten male students majoring computer science and belonging to our laboratory in our university served as participants and were divided into 2 groups of 5 students. The each group performed one of the resting actions during break times, but it did the same resting action in the mathematical experiment and the learning experiment to examine the effect of the same resting action on different types of work. In addition, we compared and discussed the learning results of this research with the results of the previous study using "aibo" and "RoBoHoN." We employed the correct-answer rate and the average number of answers as metrics for the mathematical experiment, and test score improvement as the metric for the learning experiment. We divided the learners to one of the groups through preliminary experiments so that the ability of each group is roughly equal.

\subsection{Mathematical Experiment}

The mathematical experiment is composed of three work sessions and two breaks between the work sessions. Figure 2 shows the flow of the experiment. The work session is 15 minutes long, and the resting time is 3 minutes. Figure 3 shows an example of mathematical calculation. The learner continuously calculates similar problems for 15 minutes in a work session. The mathematical experiment provides simple work in which learners tackle continuously to calculate simple numeric equations for 15 minutes. The numeric questions to be solved were produced by the automatic formula generation program of the system.

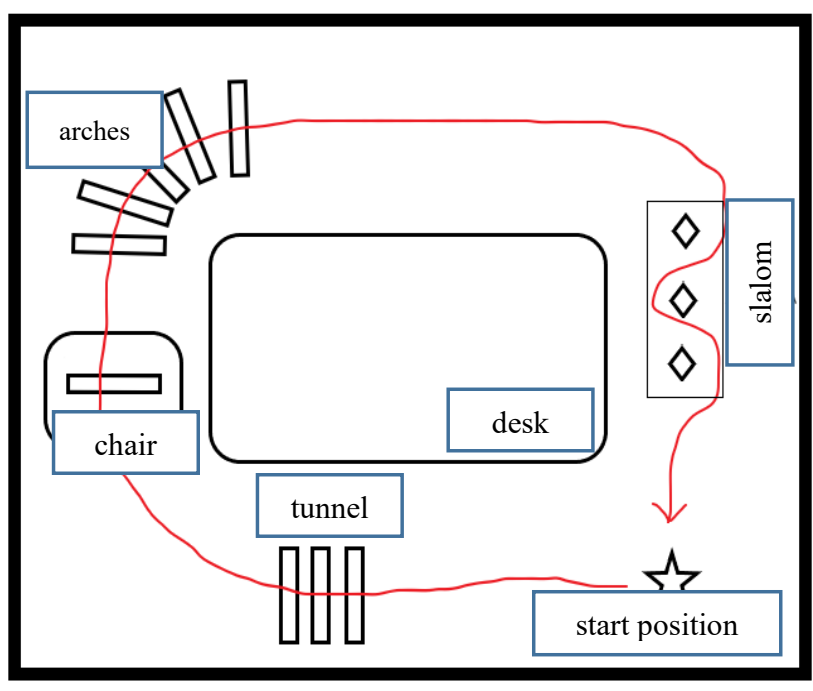

Figure 1: The basic course used for the toy drone

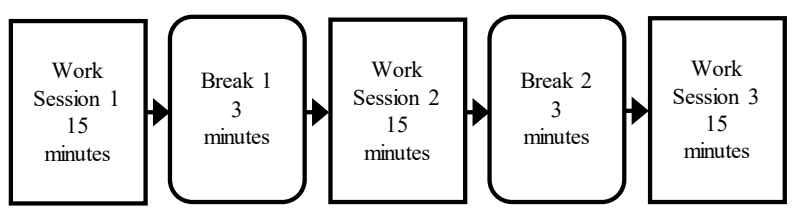

Figure 2: The flow of the mathematical experiment

Figure 4 illustrates the average correct-answer rates for the three actions. As for the aibo group, the result of the previous work [17] was incorporated with the result of the experiment in this study. From Figure 4, it can be seen that all groups tend to increase 
the correct-answer rate from the first to second work sessions and to decrease the correct-answer rate from the second to third work sessions. Among them, the drone group shows that the degree of the tendency is large, whereas that of the RoBoHoN group is small. The correct-answer rate of the aibo group behaves between the RoBoHoN group and the drone group. Considering the decrease of concentration due to fatigue in the latter half of the work sessions, the average correct-answer rate of the drone group is significantly decreased in the second to third work sessions because the resting action using the drone operations heavily needs the concentration, while the average correct-answer rate of the RoBoHoN group is gently decreased. Also, the increase of the correct-answer rate of the RoBoHoN group from the first to second work sessions is small. Regarding the aibo group, the overall average correct-answer rate is low, while the tendency is between the RoBoHoN group and the drone group. Thus, we can say that the relaxing/refreshing effect of the resting action using the drone that influences learning motivation is low, because it needs the tense concentration.

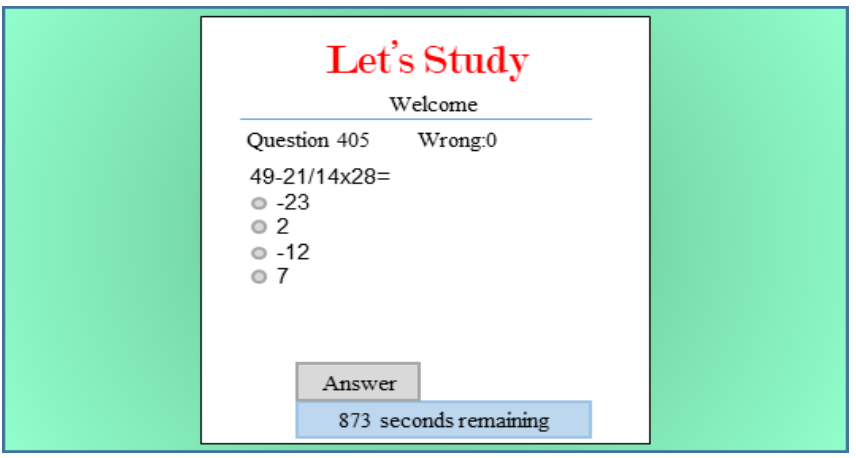

Figure 3: An example of mathematical calculation

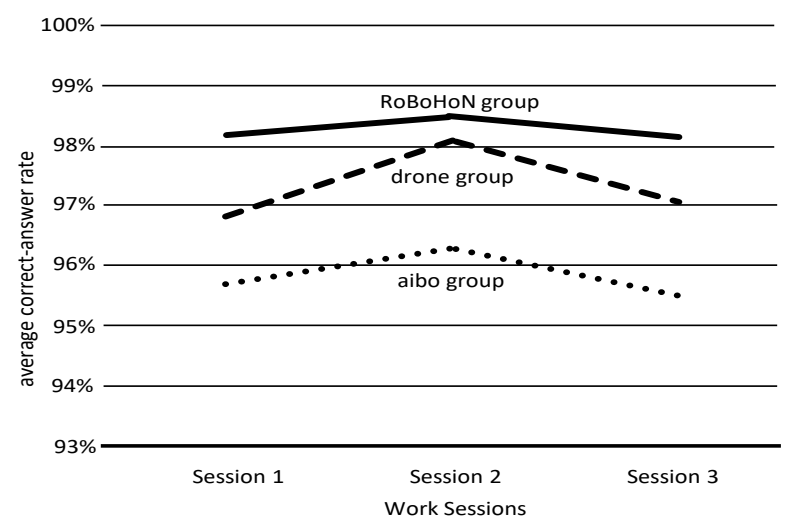

Figure 4: Correct-answer rates for RoBoHoN, aibo, and drone groups in the mathematical experiment

Figure 5 shows a graph summarizing the number of correct answers and the number of incorrect answers. Comparing the average number of answers shows that for all groups the number of answers in the second session is larger than that in the first one. Note that the increase of the number of answers by the drone group was large. However as the session proceeded, the learners became tired, and the number of answers decreased from the second to third sessions. Comparing the number of incorrect answers indicates that the numbers of the answers for all groups decreased except for the second to third sessions of the aibo group as the experiment proceeded, which suggests that the effect of the resting actions on learning was positive. Looking at Figure 5, it can be said that difference of the number of answers among the sessions is smallest for the aibo group.

In the mathematical experiment, the correct-answer rate for "playing with RoBoHoN" was a little higher than that for "playing with aibo," while the number of answers for "playing with aibo" was larger, indicating that "playing with aibo" was more effective to keep improve concentration. The authors believe that the resting behavior using aibo could provide a high refreshing/relaxing effect, and it can be considered as effective in improving learning motivation while maintaining moderate concentration.

\subsection{Learning Experiment}

In the English-word learning experiment, the learners were requested to memorize 200 English words for 30 minutes. In the experiment, two 30-minute learning sessions were held, and a 3minute break was placed between the learning sessions, as shown in Figure 6. In the experiment, we asked the learners to learn 200 English words provided by the e-learning system. A post-learning test was administered to check to see whether they memorized English words or not, immediately after the second session. An example of a question for English word learning is shown in Figure 7. Note that the group of the resting action was the same group as the mathematical experiment in order to verify the effectiveness of the resting action.

Both the pre-learning test and the post-learning test consist of 50 words. The pre-learning test was performed about a week before the experiment to prevent the learners from memorizing the English words in the test. The score of the pre-learning test was used as a baseline for comparison.

We employed the score increase rate to evaluate the learning effect of the resting actions. The increase rate was calculated as follows, where IR is the score increase rate, P1 is the score on the pre-learning test, and $\mathrm{P} 2$ is the score on the post-learning test. The full score of each test was 50 .

$$
\mathrm{IR}=\frac{P 2-P 1}{50-P 1} \times 100(\%)
$$

Figure 8 shows a graph summarizing the average increase rate of each group. We can see from Figure 8 that the RoBoHoN group had the highest average increase rate. In the memorization problem, it is necessary to remember the contents memorized in the learning sessions before the break time, and thus the resting action with high relaxation and refreshing effect is not appropriate, since the learners tend to forget the content. The authors considered that resting actions with a moderate refreshing effect is suitable. Also the positive effect was obtained for the aibo group in this experiment because the average increase rate was positive. On the other hand, the drone group has the lowest average increase rate. The resting action using the drone needs tense concentration for operating the drone, and had the high refresh effect, indicating that the resting action using the drone is not suitable.

Therefore, we can conclude that RoBoHoN-based resting action is suitable and effective for memorization learning such as English-word learning, because the score increase rate for the "playing with RoBoHoN" group was higher.

\subsection{Questionnaire and Summary}

At the time the learners finished each of the mathematical experiment and the learning experiment, we conducted a questionnaire. The questionnaire includes descriptive questions 


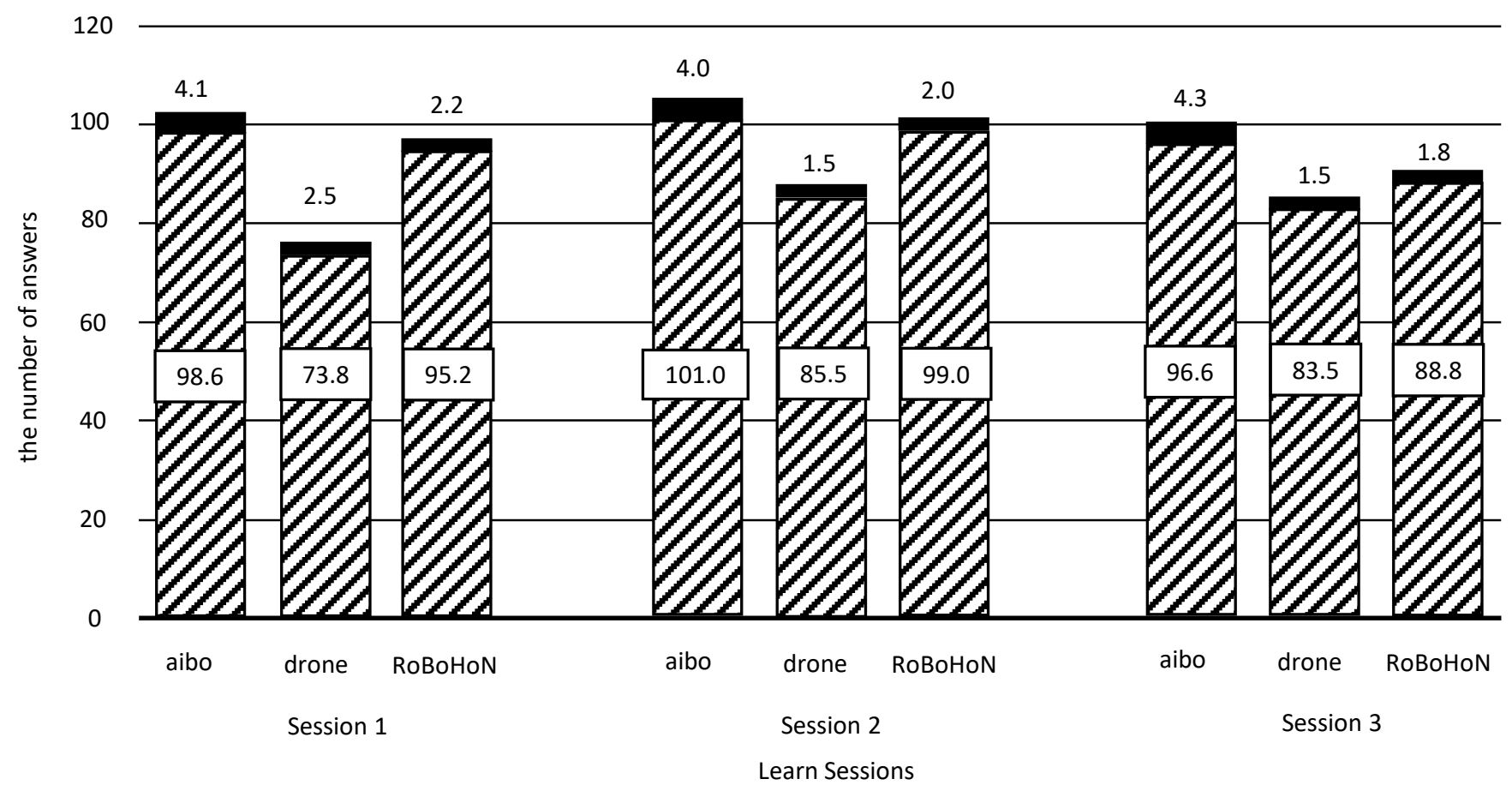

ZZZ the number of correct answers

the number of incorrect answers

Table 1: Average Scores on Survey

\begin{tabular}{|c|c|c|c|c|c|c|}
\hline \multirow{2}{*}{ Statement } & \multicolumn{2}{|c|}{ RoBoHoN group } & \multicolumn{2}{c|}{ aibo group } & \multicolumn{2}{c|}{ drone group } \\
\cline { 2 - 7 } & Math & Eng & Math & Eng & Math & Eng \\
\hline $\begin{array}{c}\text { The game-playing with the smart } \\
\text { toy during breaks were effective. }\end{array}$ & 3.8 & 3.4 & 4.1 & 4.3 & 3.2 & 2.8 \\
\hline $\begin{array}{c}\text { I felt refreshed after taking a } \\
\text { break. }\end{array}$ & 4.0 & 3.0 & 4.1 & 4.1 & 3.8 & 3.4 \\
\hline $\begin{array}{c}\text { Playing with the smart toy } \\
\text { improved my concentration. }\end{array}$ & 3.6 & 3.0 & 3.7 & 3.8 & 3.4 & 2.8 \\
\hline
\end{tabular}

and three selective questions. In the selective questions, the learners select a score on a five-point scale (5: strongly agree --- 1: strongly disagree). The questionnaire was conducted to investigate how the learners felt the effect of the resting actions.

Table 1 summarizes the evaluation results of the questionnaire obtained from aibo, drone, and RoBoHoN groups in the mathematical and the learning experiments. We can see from Table 1 that the aibo group gave the highest evaluation score for each question in both the mathematical experiment and the learning experiment, which shows higher refreshing and concentration effect than other groups did. The evaluation for the learning experiment was higher than the mathematical experiment, indicating that the resting action by aibo gave more refreshment in the learning experiment than in the mathematical experiment. We can see in Figure 5 that the number of answers was larger than those of the other groups, while in Figure 4 the correct-answer rate is the lowest, but the difference was less than 3\%. Many learners said that they regarded aibo as a robot rather than as an animal. In the limited resting time, aibo didn't behave as ordered by the learners, and that may be stressful to them. We also see in Figure
8 that the score increase rate was positive, and the resting action worked well.

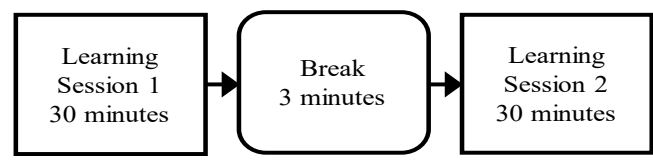

Figure 6: The flow of English-word learning experiment

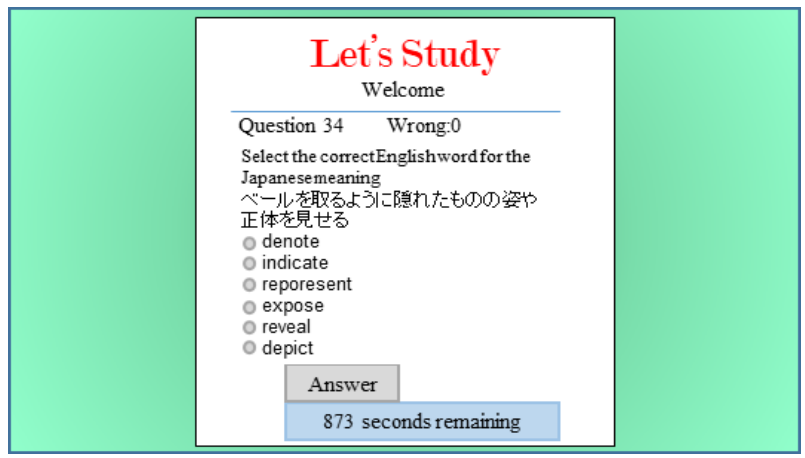

Figure 7: An example of an English-word question 


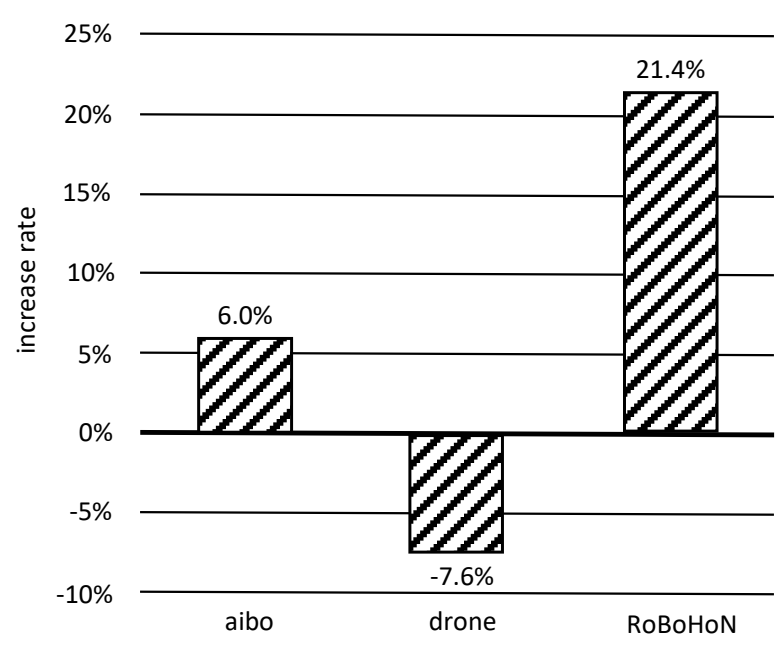

Figure 8: The average increase rates for RoBoHoN, aibo, and drone groups

The RoBoHoN group has the second highest rating scores. In this group, the evaluation of the mathematical experiment is also higher than that of the English experiments. We see in Figure 5 that the number of answers in the mathematical experiment was the second highest, while the increase rate in Figure 8 was highest in the English learning experiment, which shows that the resting action was effective in the learning experiment. The drone group has the lowest evaluation scores. They were refreshed in the mathematical experiment, while they gave evaluation score 2.8 and didn't concentrate in the learning experiment. The "playing with the drone" action needs concentration for precise operations, and that makes the learners feel stress. Thus, the authors considered that "playing with the drone" was not appropriate for the English word problem.

\subsection{Summary}

The "playing with aibo" action showed the best results as the resting action for the simple work regarding keeping and improving concentration, while the "playing with RoBoHoN" action shows the highly rated results in the English learning experiment.

The authors considered that the reason why the "playing with aibo" action was effective for the mathematical experiment was the high refreshment effect of the dog-shaped robot. The learners had to continue calculating simple mathematical problems for 15 minutes in the mathematical experiment and the "playing with aibo" highly refreshed the learners. On the other hand, in the English learning experiment, the "playing with RoBoHoN" action gained the highest score increase rate, although the evaluation scores of the "playing with aibo" group on the questionnaire were higher than those of the "playing with RoBoHoN" group. Thus, the feeling of refreshment by the learners themselves during the learning was different from the experimental results. Additionally, the "playing with RoBoHoN" action using language communication that has the moderate refreshing effect seems more suitable than the "playing with aibo" action that completely refreshed one's feeling. The "playing with a drone" behavior needs tense concentration on operation of the drone, and it was not appropriate for e-learning.

Future task includes further experiments with more participants of variety types and investigation from psychological point of view to evaluate the effect of the resting actions accurately, and the improvement of the learning system. It also includes experiments with other methods for relaxation than smart toys and discussion about the effect of those methods comprehensively in association with the type of learning.

\section{Conclusion}

In this study, we investigated the improvement of learner's concentration to learn and the learning will by using smart toys (either aibo or a toy drone) for e-learning, and discussed the effect by integrating the previous study using RoBoHoN . We compared the effect of the resting actions using aibo, RoBoHoN, and a toy drone among smart toys, since they have different features. We chose the functions that are considered to be effective for learning as the resting behavior and explained them so that the learners who touch the toys for the first time can operate the toys as they want. We found that that the "playing with aibo" action was effective for simple work like mathematical calculation, because the action had a well-balanced effect of improving concentration and learning motivation. Also we found that the "playing with RoBoHoN" action provides a moderate relaxing effect and was effective for English word memorizing. The "playing with a toy drone" action was not appropriate as a resting action, since some participants felt frustration to operate the drone.

\section{Conflict of Interest}

The authors declare no conflict of interest.

\section{Acknowledgment}

The authors would like to thank Mr. Masaki Ueta for collaboration on this work. This research was supported by a Grant-in-Aid for Scientific Research JP16K00485.

\section{References}

[1] Proseeds Inc., Merits and demerits of introducing e-learning, http://www.pro-seeds.com/trend/meritdemerit.html, Accessed in Aug. 2019

[2] A. Tominaga, C. Kogo, "Development and issues in Practical studies of elearning", The Annual Report of Educational Psychology in Japan, 53, 156165,2014

[3] M. Takeue, K. Shimada, K. Takahashi, M. Inaba, "Experiments of displaying images to keep the motivation in e-learning," Conference Proceedings IEEE International Conference on Systems, Man and Cybernetics, 120-125, 2012, doi:10.1109/ICSMC.2012.6377687.

[4] T. Ito, S. Kotake, K. Takahashi, I. Inaba, M. Inaba, "Detecting Concentration of Students Using Kinect in E-learning," 450-456, 2017, doi:10.12792/icisip2017.082.

[5] T. Ito, K. Kamiya, K. Takahashi, M. Inaba, "A method for identification of students' states using kinect," Advances in Intelligent Systems and Computing, 935, 341-350, 2019, doi:10.1007/978-3-030-19063-7_28.

[6] R. Takaoka, M. Shimokawa, T. Okamoto, "Special Section on KnowledgeBased Software Engineering A Development of Game-Based Learning Environment to Activate," 4, 911-920, 2012.

[7] A.Yamazaki, "Effect of introducing small breaks during class hours on health promotion and learning performance," Journal of humanities and sciences, Nihon University, 24(1-3), 33-58, 2019

[8] Y.Horie, "A study on optimum term of work hour with rest pause for VDT workers," Journal of Japan human factors and ergonomics society, Japan, 23(6), 373-383, 1987.

[9] T. Miki, T. Terada, et al., "Research of the effects of the break time on work performance," IPSJ SIG Technical Report, 2018-UBI-57(3), 1-8, 2018

[10] Ministry of Health, Labour and Welfare, "Guidelines for occupational health management in VDT work," http://www.mhlw.go.jp/file/06-Seisakujouhou11200000-Roudoukijunkyoku/0000184703.pdf, Accessed in Aug. 2019.

[11] D. Hirama, A. Minaduki, "A development of an interval-supported application for VDT-work," Conference of Information processing sciety of Japan, 4-125 - 4.126,2014. 
[12] M. Takeue, K. Takahashi, and M. Inaba, "Fundamental Experiments on Effects of Games in Rest Time between Learning," IPSJ SIG Technical Report, 2014-CE-124(17), 1-8, 2014 .

[13] K. Yamasaki, K. Takahashi, and M. Inaba, "Effect of the way of spending the rest time on learning," Conference of Japan Society for Fuzzy Theory and Intelligent Informatics, Kyushu Chapter, C-203, 83-86, 2015.

[14] J. Kawaguchi, T. Ito, K. Takahashi, and M. Inaba, "Influence of How to Spend Recess in E-learning," Conference of IEICE Engineering Sciences Society, A-11-4, 151, Sapporo, Japan, 2016.

[15] Y. Yasumura, T. Ito, K. Takahashi, T. Kajiyama, "Effects on Concentration of Different E-Learner Resting Behaviors in Reponse to Communication Robot Actions during Break Times," Proceedings of the 2020 14th International Conference on Ubiquitous Information Management and Communication, IMCOM 2020, 0-4, 2020, doi:10.1109/IMCOM48794.2020.9001742.

[16] L. Plowman, R. Luckin, "Interactivity, Interfaces, and Smart Toys," Computer, 37(2), 98-100, 2004, doi:10.1109/MC.2004.1266302.

[17] F.D. Faraci, M. Papandrea, A. Puiatti, S. Agustoni, S. Giulivi, V. Drapuzzo, S. Giordano, F. Righi, O. Barberis, E. Thommen, E. Rossini, “AutoPlay: A smart toys-kit for an objective analysis of children ludic behavior and development," MeMeA 2018 - 2018 IEEE International Symposium on Medical Measurements and Applications, Proceedings, 2018, doi:10.1109/MeMeA.2018.8438636.

[18] S. Shasha, M. Mahmoud, M. Mannan, A. Youssef, "Playing with danger: A taxonomy and evaluation of threats to smart toys," IEEE Internet of Things Journal, 6(2), 2986-3002, 2019, doi:10.1109/JIOT.2018.2877749.

[19] Sharp Corporation, "RoBoHoN," http://global.sharp/corporate/news/160414.html, Accessed in Aug. 2019.

[20] Sony corporation, "aibo," https://us.aibo.com/, Accessed in Aug. 2019.

[21] Mail Online, "The power of cute: How looking at pictures of baby animals can help improve your concentration levels," https://www.dailymail.co.uk/news/article-2210614/The-power-cute-Howlooking-pictures-baby-animals-help-improve-concentration-levels.html, Accessed in Aug. 2019.

[22] Ryze Technology Co.,Ltd, "Tello," https://www.ryzerobotics.com/jp/tello/, Accessed in Aug. 2020. 